\title{
Pharmacodynamic study of the selenium-mediated arsenic excretion in arsenicosis patients in Bangladesh
}

\author{
M. Alauddin ${ }^{1}$, R. Cekovic ${ }^{1}$, S. Alauddin ${ }^{1}$, L. Bolevic ${ }^{1}$, S. Saha ${ }^{2}$, J.E. Spallholz ${ }^{3}$, P.F. LaPorte ${ }^{4}$, \\ S. Ahmed ${ }^{5}$, H. Ahsan ${ }^{6}$, J. Gailer ${ }^{7}$, O. Ponomarenko ${ }^{8}$, I.J. Pickering ${ }^{8}$, S.P. Singh ${ }^{8} \&$ G.N. George ${ }^{8}$ \\ ${ }^{1}$ Department of Chemistry, Wagner College, Staten Island, New York, USA \\ ${ }^{2}$ BIRDEM Hospital, Dhaka, Bangladesh \\ ${ }^{3}$ Division of Nutritional Sciences, Texas Tech University, Lubbock, Texas, USA \\ ${ }^{4}$ Division of Hematology-Oncology, University of California Los Angeles, Los Angeles, California, USA \\ ${ }^{5}$ Institute of Child and Mother Health, Sk Hospital, Dhaka, Bangladesh \\ ${ }^{6}$ Department of Health Sciences, University of Chicago, Chicago, USA \\ ${ }^{7}$ Department of Chemistry, University of Calgary, Calgary, Canada \\ ${ }^{8}$ Department of Geological Sciences, University of Saskatchewan, Canada
}

\begin{abstract}
Since 1938 several investigators have confirmed antagonistic interaction whereby the administration of selenite mitigates arsenite toxicity in mammals. More recently a number of animal model and human studies have revealed that the bimolecular basis of this antagonism involves the glutathione mediated formation of major metabolite seleno-bis(S-glutathionyl)arsinium [(GS)2AsSe]-ion which is rapidly excreted through bile. To investigate whether the oral supplementation of sodium selenite will enhance the fecal excretion of arsenites in human, a limited pharmacodynamics study was carried out involving ten arsenicosis patients. However, data from five patients are reported here. Patients were screened for pre-existing hepatic diseases and they were excluded from the study. Subjects were selected based on the arsenic level in their household drinking water, hair, nail samples and diffuse melanosis symptoms. Patients received ${ }^{77} \mathrm{Se}-l a b e l e d$ sodium selenite as oral supplement, which they ingested together with their arsenic-containing drinking water. Total selenium levels increased in both urine and feces following the co-administration of sodium selenite $(800 \mu \mathrm{g})$. The patients receiving a placebo showed no change in their selenium excretion. The increase in selenium levels in feces and arsenic to some extent after dosing agrees with our hypothesis that selenium supplementation promotes co-excretion of arsenic and selenium through formation of [(GS)2AsSe]- in the bile.
\end{abstract}

\section{INTRODUCTION}

Since the discovery of unsafe levels of arsenic in groundwater in Bangladesh in 1993, a number of reports have indicated that approximately 70-80 million people are being exposed to this metalloid to concentrations in drinking water that are above the World Health Organization (WHO) permissible level of $10 \mu \mathrm{g} \mathrm{L}^{-1}$ from shallow aquifers contaminated by geogenic arsenic (Mukherjee \& Bhattacharya, 2001; Kinniburgh \& Smedley 2001; Ahmed et al., 2006). Chronic exposure of humans to inorganic arsenic leads to arsenicosis with pathological symptoms including skin melanosis and keratosis, endocrine diseases and cancers of the lungs, the liver, the kidneys and the bladder (Kapaj et al., 2006).

\subsection{Antagonism of Arsenic and selenium}

During 1930-1950, several animal model studies indicated that arsenite can detoxify selenium in form of selenite and/or seleniferous wheat in rats, dogs, cattles (Moxon et al., 1938). The therapeutic benefit of selenium for countering the toxicity of inorganic arsenic stems from a number of studies that have been carried out over the past 80 years. The oxy-anions of arsenic and selenium are arsenite and selenite - are toxic to animals if given separately. However, if both are administered together they mutually de-toxify each other. This antagonistic interaction has been linked to the in vivo formation of the seleno-bis(S-glutathionyl)arsinium [(GS)2AsSe]-. Synchrotron-based X-ray absorption (XAS) spectroscopy has recently revealed the formation of the seleno-bis(S-glutathionyl)arsinium anion in hepatocytes of rabbits and its subsequent excretion in the bile (Gailer et al., 2002, 2004). In addition, studies indicate that multidrug resistance protein 2 (MRP2) transports [(GS)2AsSe]- mediates its excretion from the liver to the bile (Carew \& Leslie, 2010).

During 2006 to 2009 we have conducted a 48 -week, 821 patient, randomized, double-blinded placebo controlled Phase III clinical trial in Bangladesh to 
investigate the effectiveness of daily oral dose of $200 \mu \mathrm{g}$ selenite in combating arsenic toxicity. Our study suggested that the employed selenium dose was inadequate to reduce the prevalent condition of melanosis and other arsenicosis symptoms since their arsenic intake through drinking water and food were quite high (Alauddin et al., 2012). However, our recent studies which involved the administration of selenite and arsenite to rats and hamsters conclusively revealed the presence of [(GS)2AsSe]- in the bile (George et al., 2016; Ponomarenko et al., 2017).

\subsection{Objectives of the current study}

A number of our previous studies using rabbits, rats, hamsters and human have demonstrated that the coadministration of selenite will promote the hepatobiliary co-excretion of arsenic and selenium in the form of the $[(\mathrm{GS}) 2 \mathrm{AsSe}]-$ ion. We hypothesize that the dietary supplementation of selenium may promote the formation of the arsenic-selenium-glutathione conjugate which is excreted in the bile and therefore increases the excretion of arsenic via feces. The present study is a follow up is a follow up of the previous pharmacodynamics study involving five patients. Our objectives were to monitor the excretion of total arsenic and selenium via feces and urine in five patients that were provided with daily meals and drinking water which they would normally consume in their household. Our aim was to assess the effectiveness of a selenium supplement in the excretion of arsenic from the body of arsenicosis patients.

\section{EXPERIMENTAL}

\subsection{Study design}

This is a limited study which involved five arsenicosis patients from Laksham, Shahrasty in Bangladesh. The subjects were patients which were recruited following defined selection criteria including a) arsenic exposure based on arsenic in their drinking water source, and b) normal kidney and liver function. The study protocol was explained to all patients and signed consents was obtained from all participants. All participants stayed in a private clinic in Laksham for 10 days where they were given fixed food regimen prepared with the drinking water from their homes. On the sixth day they received either a $800 \mu \mathrm{g} \mathrm{Se}-77$ labeled sodium selenite dose or a placebo, which was drinking water that did not contain selenite. The stable isotope Se-77 labelled sodium selenite was used as supplement to differentiate native selenium and selenium that was administered to patients. All urine and fecal matter were collected from all participants every day to account for all excretions. Feces were weighed, volumes of urine were measured and all excretions were recorded during the patients 10 day stay in the clinic. Data for 5 th thru 8th day are presented in this paper. The urine samples and fecal matters were pooled together separately for each day for each participant. All food samples for breakfast, lunch, dinner were weighed for each participant every day and recorded. A portion of breakfast, lunch, dinner, snacks were homogenized in distilled deionized water and aliquots were preserved in vials for arsenic and selenium analysis. All water, fruit juice, tea intake for each patient were recorded daily. All pooled feces samples were weighed and an weighed portion was homogenized in distilled deionized water and aliquots were preserved at $-4^{\circ} \mathrm{C}$ in a freezer before further analysis in the laboratory. The aliquots of food, water, patient samples such as blood, urine and feces were transported to the USA and stored immediately in a $-80^{\circ} \mathrm{C}$ freezer prior to analysis.

\subsection{Reagents and standards used for the chemical analysis of all biological tissues for total arsenic and selenium}

Homogenized feces samples were oven dried at $95^{\circ} \mathrm{C}$ for an hour and dry weight was obtained. The dried fecal matter were digested in high purity nitric acid and diluted with deionized water to a fixed volume. Arsenic (As), selenium (Se) in urine and fecal matter were determined by the DRC-ICP-MS technique while the arsenic, selenium in food and water samples were determined by the graphite furnace atomic absorption spectroscopy (GF-AAS) (Perkin Elmer Model AAnalyst 800) with Zeeman background correction and electrodeless discharge lamps (EDL). Matrix modifier for $\mathrm{Se}$ analysis were prepared from a solution containing $1 \%(\mathrm{w} / \mathrm{v}) \mathrm{Ni}\left(\mathrm{NO}_{3}\right)_{2} \cdot 6 \mathrm{H}_{2} \mathrm{O}, 2 \%$ (w/v) $\mathrm{Mg}\left(\mathrm{NO}_{3}\right)_{2} \cdot 6 \mathrm{H}_{2} \mathrm{O}$ and $0.1 \%(\mathrm{v} / \mathrm{v})$ Triton $\mathrm{x}-100$ while the matrix modifier for As analysis consisted of $1 \%(\mathrm{w} / \mathrm{v}) \mathrm{Ni}\left(\mathrm{NO}_{3}\right)_{2} .6 \mathrm{H}_{2} \mathrm{O}$ and $0.1 \%$ Triton $\mathrm{X}$ 100 . For all matrix modifiers, high purity $(99.999 \%)$ $\mathrm{Ni}\left(\mathrm{NO}_{3}\right)_{2} \cdot 6 \mathrm{H}_{2} \mathrm{O}, 2 \%$ (w/v) $\mathrm{Mg}\left(\mathrm{NO}_{3}\right)_{2} \cdot 6 \mathrm{H}_{2} \mathrm{O}$ (SigmaAldrich Co., USA) $18 \mathrm{M} \Omega \mathrm{cm}^{-1}$ water were used. Stock As, Se standard solutions (Perkin Elmer Co., USA) were used to calibrate AAS. The National Institute of Standards and Technology (NIST) standard reference materials (SRM 1640), served as control reference material for As, Se analysis in water and food samples. The method detection limit for As, Se analysis in water and urine was $1.0 \mu \mathrm{g} \mathrm{L}^{-1}$, with an RSD of $2.5 \%$.

\section{RESULTS}

\subsection{Fecal and urinary $\mathrm{Se}$, As data}

Arsenic and selenium in fecal matter from all (5) patients collected before Se dosing and after Se dosing are shown in Table 1. Patients 1, 2 and 3 received selenium supplements and patients 4 and 5 received placebo (drinking water without selenium dose) on day 6 . Here we report data for day 5 thru day 8 for brief observation for the fate of selenium administered to patients. 
Table 1. Daily As, Se intake and pooled fecal and urinary excretion $(\mu \mathrm{g})$ by patients .

\begin{tabular}{|c|c|c|c|c|c|c|c|}
\hline & & In & Ex & In & Ex & In & Ex \\
\hline & Day & As & As & $\mathrm{Se} 78$ & $\mathrm{Se} 78$ & Se77 & Se77 \\
\hline \multirow[t]{4}{*}{ Pat1 } & 5 & 2026 & 1825 & 39.4 & 53.9 & 0 & 74.6 \\
\hline & 6 & 1948 & 1688 & 24.2 & 49.8 & 800 & 54.6 \\
\hline & 7 & 2161 & 1221 & 30.7 & 50.1 & 0 & 9985 \\
\hline & 8 & 1803 & 1224 & 19.7 & 46.6 & 0 & 4132 \\
\hline \multirow[t]{4}{*}{ Pat2 } & 5 & 1400 & 374 & 39.6 & 21 & 0 & 23.3 \\
\hline & 6 & 1146 & 602 & 23.9 & 30.5 & 800 & 31.9 \\
\hline & 7 & 1488 & 497 & 38.7 & 31.3 & 0 & 4352 \\
\hline & 8 & 1401 & 877 & 19.4 & 69.4 & 0 & 7328 \\
\hline \multirow[t]{4}{*}{ Pat3 } & 5 & 1846 & 694 & 39.4 & 27.7 & 0 & 31.9 \\
\hline & 6 & 1906 & 1237 & 26.5 & 38.9 & 800 & 44.6 \\
\hline & 7 & 1771 & 391 & 31.9 & 17.2 & 0 & 4318 \\
\hline & 8 & 1835 & 732 & 19.7 & 16.6 & 0 & 1995 \\
\hline \multirow[t]{4}{*}{ Pat4 } & 5 & 1804 & 2435 & 59.9 & 52.8 & 0 & 56.6 \\
\hline & 6 & 1774 & 1853 & 35.9 & 52.4 & 0 & 57.6 \\
\hline & 7 & 1767 & 1199 & 64.2 & 32.8 & 0 & 63.1 \\
\hline & 8 & 1801 & 1958 & 23.8 & 77.9 & 0 & 88.5 \\
\hline \multirow[t]{4}{*}{ Pat5 } & 5 & 1344 & 646 & 38.9 & 25.6 & 0 & 26.6 \\
\hline & 6 & 1210 & 852 & 23.4 & 29.4 & 0 & 33.7 \\
\hline & 7 & 1416 & 632 & 37.2 & 25.8 & 0 & 28.7 \\
\hline & 8 & 1634 & 867 & 19.4 & 33.9 & 0 & 46.2 \\
\hline
\end{tabular}

\section{As and Se intake and excretion data $(\mu \mathrm{g})$}

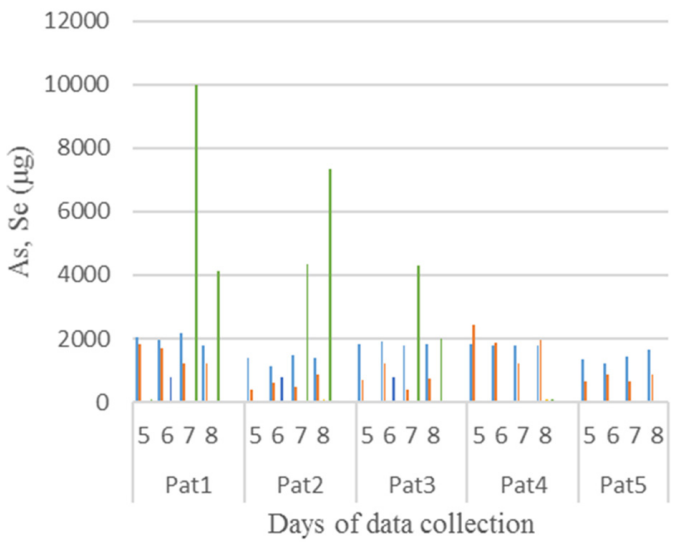

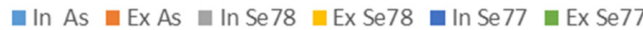

Figure 1. As, Se intake and excretion $(\mu \mathrm{g})$ for patients 1-5.

\subsection{Intake and excretion of As, Se}

In Table 1 total As, Se intake by a patient are calculated from the As, Se levels in their daily food intake and their drinking water consumed by each patient. The total excretion of As, Se have been determined from the As, Se levels in urine and fecal matter and the pooled amount of urine and fecal matter for each patient for each day. Patients 1-3 received Se supplementation on day 6 . Patients 4 and 5 did not receive selenium supplement.

The As and Se in fecal excretion are high, especially in fecal matter for the supplement group (Table 1) as
Arsenic, Selenium excretion in fecal matter

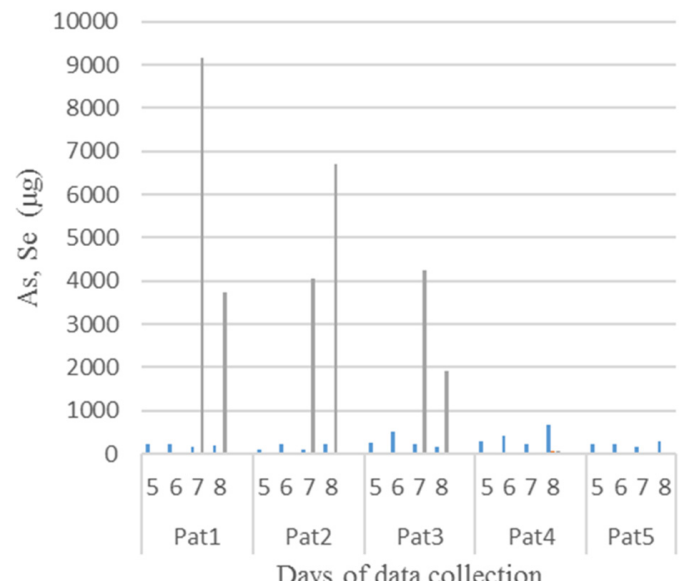

Figure 2. Fecal excretion of total As and Se by patients.

Arsenic, Selenium excretion in urine

2500

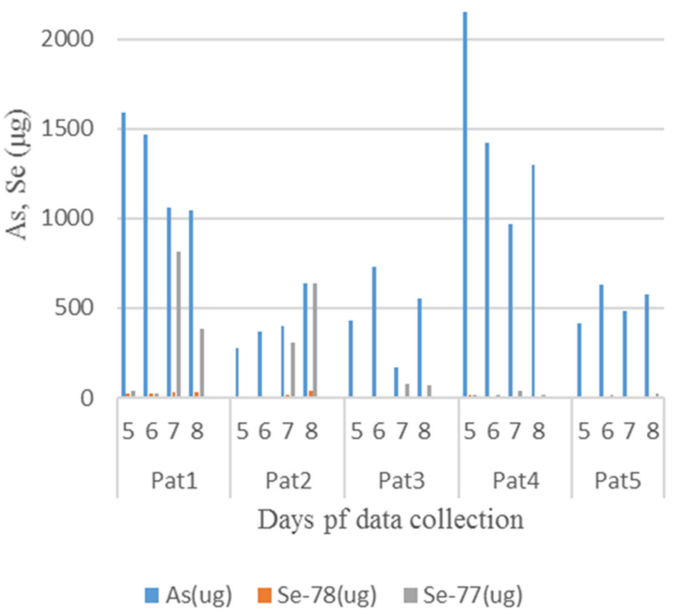

Figure 3. Urinary excretion of total As and Se by patients.

expected. Data for only 2 pre-dose days and 2 postdose days have been reported here for a brief overview of the pharmacodynamics study. Detailed outcome involving all ten patients and analysis of patient samples from all 10 days will be reported later. The present data indicate that (a) selenium increases in fecal matter in supplement group on the 7th and 8th days at the appropriate interval 6-12 hours after Se ingestion and (b) in every case fecal arsenic excretion increases after Se ingestion. The total amount of Se-77 excretion is much higher in all three patients in the supplement group than the intake amount. The urinary As, Se excretion increased for patient 2, 3 in the supplement group. High urinary As excretion in patient 4 might 
be indicative of other physiologic condition for this patient.

\section{CONCLUSIONS}

The urine, fecal matter samples from the remaining 5 patients in the same batch are being analyzed and the data will be reported later in a separate paper. The current data obtained from a limited number of patients are indicative of potential pathway for removal of body burden arsenic by Se supplementation. Currently a pharmacodynamics study involving a larger number of patients is under way by our group.

\section{CLINICAL TRIAL REGISTRY}

Clinical Trial.gov Identifier: NCT02377635.

\section{ACKNOWLEDGEMENTS}

The study has been supported by grant from Grand Challenges Canada to the University of Saskatchewan. The authors thank R. Gerads from the Brooks Applied Laboratory, Bothell, Washington, USA for Se measurements in samples through ICP-MS.

\section{REFERENCES}

Ahmed, M.F., Ahuja, S., Alauddin, M., Hug, S.J., Lloyd, J.R., Pfaff, A., Pichler, T., Saltikov, C., Stute, M. \& van Geen, A. 2006. Epidemiology: ensuring safe drinking water in Bangladesh. Science 314: 1687-1688.

Alauddin, M., Wheaton, T., Valencia, M., Stekolchik, E., Spallholz, J.E., La Porte, P.F., Ahmed, S., Chakaraborty, B., Bhattacharjee, M., Zakaria, A.B.M., Sultana, S., George, G.N., Pickering, I.J. \& Gailer, J. 2012. Clinical trial involving selenium supplementation to counter arsenic toxicity among rural population in Bangladesh In: J.C. Ng, B.N. Noller, R. Naidu, J. Bundschuh \& P. Bhattacharya (Eds.) Understanding the Geological and Medical Interface of
Arsenic As 2012: Proceedings of the 4th International Congress on the Arsenic in the Environment. Taylor and Francis Group, CRC Press, London, UK. pp. 143-147.

Carew, M.W. \& Leslie, E.M. 2010. Selenium-dependent and independent transport of arsenic by human multidrug resistance protein 2 (MRP2/ABCC2): implications for the mutual detoxification of arsenic and selenium. Carcinogenesis 31: 1450-1455.

Gailer, J., George, G.N., Pickering, I.J., Prince, R.C., Younis, H.S. \& Winzerling, J.J. 2002. Biliary excretion of $\left[(\mathrm{GS})_{2} \mathrm{AsSe}\right]^{-}$after intravenous injection of of rabbits with arsenite and selenite. Chem. Res. Toxicol. 15: 1466-1471.

Gailer, J., Ruprecht, I., Reitmeir, P., Benker, B. \& Schramel, P. 2004. Mobilization of exogenous and endogenous selenium to bile after the intravenous administration of environmentally relevant doses of arsenite to rabbits. Appl. Organomet. Chem. 18: 670-675.

George, G.N., Gailer, J., Ponomarenko, O., La Porte, P.F., Strait, K., Alauddin, M., Ahsan, H., Ahmed, S., Spallholz, J.E. \& Pickering, I.J. 2016. Observation of the selenobis-(S-glutathionyl) arsinium anion in rat bile. Inorg. Biochem 158: 24-29.

Kapaj, S., Peterson, H., Liber, K. \& Bhattacharya, P. 2006. Human health effects from chronic arsenic poisoning-a review. J. Environ. Sci. Health Part A 41(10): 2399-2428.

Kinniburgh, D.G. \& Smedley, P.I. (Eds) 2001. Arsenic contamination of groundwater in Bangladesh, Final Report (BGS Technical Report WC/00/19, British Geological Survey, Keyworth, UK.

Mukherjee, A.B. \& Bhattacharya, P. 2001. Arsenic in groundwater in the Bengal delta Plain, slow poisoning in Bangladesh. Environ. Res. 9(3): 189-220.

Moxon, A.L. 1938. The effect of arsenic on the toxicity of seleneferous grains. Science 88, 81.

Ponomarenko, O., La Porte, P.F., Singh, S.P., Langan, G., Fleming, D.E.B., Spallholz, J.E., Alauddin, M., Ahsan, H., Ahmed, S., Gailer, J., George, G.N. \& Pickering, I.J. 2017. Selenium-mediated arsenic excretion in mammals: a synchrotron-based study of whole-body distribution and tissue-specific chemistry. Metallomics 9: 1585-1595. 